\title{
Cue-framing effects in source remembering: A memory misattribution model
}

\author{
IAN G. DOBBINS \\ Washington University, St. Louis, Missouri \\ AND \\ DANIEL MCCARTHY \\ Duke University, Durham, North Carolina
}

\begin{abstract}
During source remembering, selectively emphasizing one source in the retrieval query "Is this item from Source A?" can yield different accuracy than emphasizing the alternate source in "Is this item from Source B?" even if those are the only two possible origins. One account of this cue-framing effect holds that it reflects different active monitoring strategies encouraged by the two cue frames. An item memory misattribution (IMM) model instead assumes that this effect reflects the uncontrolled use of item recognition during confirmatory source judgments, and an IMM model simulation predicted a quantitative relationship between recognition levels and the cue-framing effect. Experiments 1 and 3 confirmed these predictions by using study repetitions to manipulate recognition levels, and Experiments 2 and 3 also demonstrated the effect with new source tasks not previously considered. The data suggest that, in addition to qualitative monitoring strategies, subjects also use the availability of item memory in a heuristic fashion during confirmatory source attributions.
\end{abstract}

In an everyday environment, we often try to remember the prior origin of people or things that strike us as familiar, and the evolutionary benefit of being able to remember whether a particular stimulus was previously encountered under beneficial or detrimental circumstances is self-evident. In the experimental literature, this skill is typically referred to as source memory. During source memory tasks, subjects are usually presented with probes drawn from two prior encoding contexts, and their task is to determine from which prior source context each probe originated. The context attributes that have been examined have been varied, with some related to the prior appearance of the probes (e.g., previous font color), their prior positioning (e.g., left or right side of screen), or the types of cognitive operations previously performed on the probes (e.g., living/nonliving or pleasant/unpleasant judgments).

One method of characterizing source memory performance is as a one-dimensional signal detection process (e.g., Qin, Raye, Johnson, \& Mitchell, 2001; Slotnick \& Dodson, 2005). When applied to source memory data, the model assumes that the source items evoke two continuous, overlapping normal evidence distributions. The distance between the modes of the distributions, $d^{\prime}$, indicates the sensitivity of the memory system to the context memory distinction that operationally defines the sources. As the evidence triggered by a given probe becomes extreme in either direction, observers will have more confidence that the item originated from one versus the other source. However, because the evidence is overlapping, observers are forced to use a particular evidence value, termed a decision criterion, to parse the continuum into two categorical response regions (e.g., "Source A" or "Source B"). When confidence is also required, it is simply assumed that additional decision criteria are used to parse the continuum into a larger number of response categories (for reviews, see Banks, 1970; Macmillan \& Creelman, 1991; T. E. Parks, 1966). If the decision model is correct, then one can estimate the accuracy of observers completely independently of their chosen criterion placement.

Under the one-dimensional signal detection model, it is assumed that the memory evidence remains stationary during manipulations that shift the decision criterion. Indeed, this is arguably the fundamental assumption of signal detection theory, since if it is violated the goal of estimating accuracy independently of decision criteria becomes impossible. Given the assumption of stationary memory evidence, researchers typically assume that experiments using slightly different response methods nonetheless yield comparable accuracy results. For example, recognition research reports often differ in whether they require graded confidence judgments or, instead, simply require subjects to make a dichotomous old/new distinction. Furthermore, within confidence rating tasks, there is a wide range of scalings used across studies. The fact that researchers often jointly consider these experiments during theory building speaks to the widely held belief that the data across these studies are comparable, which in turn reflects the assumption that minor response for- 
mat changes do not systematically affect the nature of the memory evidence across studies.

Although there do not appear to be data that put in question the assumption of stationary evidence across mild response cue changes in item recognition, evidence violating this assumption during source memory has been provided in an important study by Marsh and Hicks (1998). In Experiment 1 of their study, subjects either generated solutions for anagrams or simply read words that were presented intact. Later, during source memory testing for probes drawn from these two contexts, subjects were prompted with the retrieval cue "Did you see?" or its complementary form, "Did you generate?," when they attempted to discriminate the source probes. Given the one-dimensional signal detection model in Figure 1, there is little a priori reason to assume that this minor manipulation of the retrieval cue would affect accuracy $\left(d^{\prime}\right)$, although one might predict that it could have an influence on the placement of the decision criterion. However, the researchers found that the framing of the cue had a significant effect on source discrimination accuracy, with the cue frame highlighting generation ("Did you generate?") leading to a clear accuracy advantage over the frame mentioning seeing (Marsh \& Hicks, 1998). We refer to this accuracy difference as a cue-framing accuracy effect and to the different forms of the cue as different cue frames.
Marsh and Hicks (1998) noted that the cue-framing effect posed potential difficulties for the basic signal detection account and offered an interpretation based instead on the source monitoring framework of Johnson and colleagues (Johnson, Hashtroudi, \& Lindsay, 1993). More specifically, they suggested that different cue frames lead subjects to monitor or search memory differently. With the "Did you generate?" cue frame, it was assumed that subjects monitored memory for evidence of prior cognitive operations that would have been recruited when previously encountering the item. In contrast, it was assumed that the "Did you see?" frame led subjects to instead monitor memory for the prior perceptual characteristics of the probes. Marsh and Hicks argued that if memory for prior cognitive operations is more available or diagnostic than memory for visual details, cue-framing effects would occur because the "Did you see?" frame would lead to a strategy that is less effective in recovering this useful content. We refer to this explanation as the strategic monitoring account because it hinges on the hypothesis that cue frames cause subjects to shift between more or less effective memory monitoring strategies. In support of this model, when the two encoding contexts were seeing or hearing the to-be-encoded items, later source discrimination accuracy of the studied items was unaffected by the framing of the cue (i.e., Did you see? vs. Did you hear?; Experiment 2). ${ }^{1}$ From the strategic monitoring per-

\section{From Source A? Yes or No}

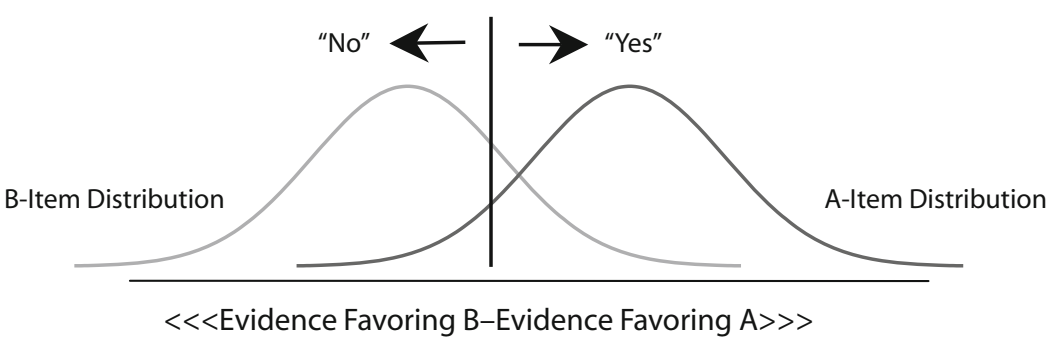

From Source B? Yes or No

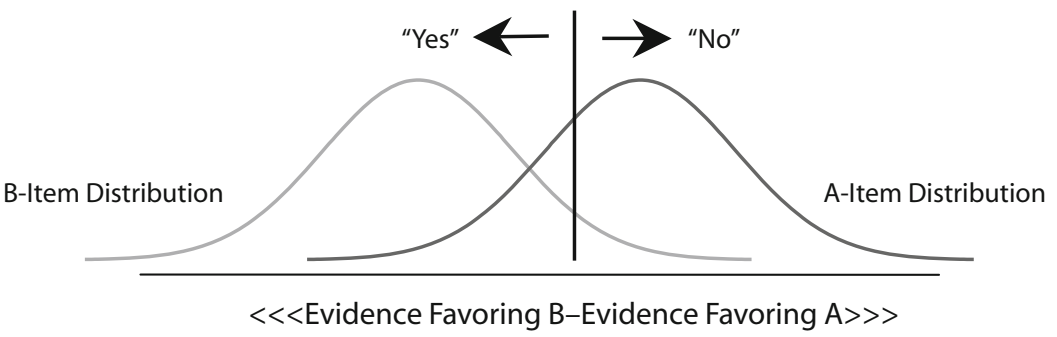

Figure 1. Illustration of a one-dimensional signal detection model of source discrimination. Items from two sources are assumed to yield normal evidence distributions separated by a distance $\left(d^{\prime}\right)$. The relative order of the item distributions along the axis is arbitrary. Evidence favoring one or the other source accrues as the values become extreme in either direction. In the top panel, when the source cue reads "From Source A?" observers will respond "yes" when the evidence in the A direction exceeds a decision criterion indicated by the vertical line. On the bottom panel, when the question is instead framed "From Source B?" observers will assign "no" responses to items above the criterion. The illustration assumes that although the nature of the evidence remains unchanged by the framing of the cue, the decision criterion shifts in a manner that favors affirmative responding under each cue frame. 
spective, this null effect was expected, since both frames should encourage observers to adopt similarly effective visual or auditory perceptual monitoring strategies. If one assumes that mnemonic perceptual information is equally available for seen and for heard items, then switching of strategies would not lead to differences in measured source discrimination accuracy.

In the present study, we extend the findings of Marsh and Hicks (1998) and also test a complementary account of cue-framing effects referred to as the item memory misattribution, or IMM, model. Under this account, the difference in accuracy for the different source memory cue frames can result from the tendency of subjects, when source recollection fails or is limited, to inappropriately take item memory as supportive of the source framed by the cue. That is, item memory is assumed to generally "push" or "bias" subjects toward the affirmative response framed by the cue, even if what is recovered does not uniquely relate to the dichotomous source distinction implied by the question. Under the model, if item memory systematically differs for the probes drawn from the two examined sources, cue-framing accuracy differences will naturally emerge. Conceptually, the IMM model is similar to a trial-specific availability or similarity heuristic (Gilovich, Griffin, \& Kahneman, 2002) during source memory attributions. More specifically, considerable work in judgment and decision-making has suggested that subjects judge the likelihood of propositions or events in proportion to the ease with which they can retrieve instances that are similar to those propositions or events. In the case of source attributions, then, the idea is that the cue (e.g., "Did you generate?") is treated as a proposition with a particular likelihood of being true given a memory probe. The IMM model assumes that the subject not only uses diagnostic content when available, but in the absence of such content, mistakenly accepts as diagnostic the availability of memory content that generally satisfies the gist of the experience.

\section{Model Equations and Simulation}

The IMM model makes two basic assumptions. The first is that test items can evoke episodic information that is both diagnostic (d) and nondiagnostic (nd) with respect to a specific source memory distinction. For example, if subjects are asked to determine the prior spatial location of test probes (left or right), the model assumes that in addition to this diagnostic location information, subjects may also recover other contextual details regarding the previous encounter, such as prior thoughts, item attributes, or other event characteristics associated with the probe's prior appearance. Although these remembrances are not specifically unique to the required contextual distinction, they nonetheless are generally consistent with the prior encounter in the experiment. This type of recollection has also been referred to as noncriterial recollection in the context of dual-process studies of recognition (for a brief review, see C. M. Parks, 2007). In addition, the item may evoke acontextual feelings of familiarity, which are likewise nondiagnostic for the current context memory query, in the sense that subjects should not have any a priori reason to believe that item familiarity should systematically differ across the relevant sources. It is important to note that the model does not require one to assume a strong process distinction between these two putative types of memory processes (contextual recollection vs. item familiarity), and here both are simply grouped under the label nondiagnostic item memory because they do not uniquely relate to the specific source distinction of interest.

The second key assumption of the model is that in the absence of diagnostic context information, subjects are positively influenced or biased by nondiagnostic item memory content toward affirmation of the cue frame; that is, they are assumed to be increasingly more willing to respond "yes" to the source memory query as general recognition of the item itself becomes more vivid or certain. This assumption is strongly supported by research in numerous recognition domains that has demonstrated that subjects are prone to the influence of item information, even when the information is ostensibly nondiagnostic with respect to a current context memory demand (e.g., Jacoby, Kelley, Brown, \& Jasechko, 1989; Jacoby, Woloshyn, \& Kelley, 1989; Kelley \& Wixted, 2001; Koriat \& Levy-Sadot, 2001; Lindsay, Pozzulo, Craig, Lee, \& Corber, 1997; Whittlesea, Jacoby, \& Girard, 1990). Given these two basic assumptions, one can more formally estimate the response probabilities for two hypothetical source item types (A and B) under two different cue frames ("Source A?" and "Source B?") and see whether the IMM model in fact predicts cue-framing accuracy effects. Under the "Source A?" cue frame, the probabilities of correct and incorrect "yes" responses are as follows:

$$
\begin{aligned}
& \text { "Source A?" Query } \\
& P(\text { "yes" } \mid \mathrm{A} \text { item })=\mathrm{A}_{\mathrm{d}}+\left(1-\mathrm{A}_{\mathrm{d}}\right) \mathrm{A}_{\text {nd }}=\text { "hit," } \\
& P(\text { "yes" } \mid \mathrm{B} \text { item })=\left(1-\mathrm{B}_{\mathrm{d}}\right) \mathrm{B}_{\mathrm{nd}}=\text { "false alarm." }
\end{aligned}
$$

Equation 1 indicates that correct "yes" responses to A items under the "Source A?" cue frame (hits) will occur if diagnostic contextual information is recovered $\left(A_{d}\right)$ or if, in its absence $\left(1-A_{d}\right)$, nondiagnostic information is sufficiently strong to provoke a "yes" response $\left(A_{n d}\right)$. In the case of items drawn from the B source, Equation 2 indicates that incorrect "yes" responses under the "Source A?" frame (false alarms) will occur only if the subject fails to recover sufficient diagnostic information that the item is from the inappropriate source $\left(1-\mathrm{B}_{\mathrm{d}}\right)$ and if nondiagnostic item information is sufficiently strong to provoke a "yes" response $\left(\mathrm{B}_{\mathrm{nd}}\right)$. In the case of the "Source B?" cue frame, the equations are, analogously:

$$
\begin{aligned}
& \text { "Source B?" Query } \\
& P(\text { "yes" } \mid \mathrm{B} \text { item })=\mathrm{B}_{\mathrm{d}}+\left(1-\mathrm{B}_{\mathrm{d}}\right) \mathrm{B}_{\mathrm{nd}}=\text { "hit," } \\
& P(\text { "yes" } \mid \text { A item })=\left(1-\mathrm{A}_{\mathrm{d}}\right) \mathrm{A}_{\mathrm{nd}}=\text { "false alarm." }
\end{aligned}
$$

From these equations, it becomes clear why systematic differences in the levels of nondiagnostic item memory can lead to cue-framing source accuracy effects. For example, if one assumes that diagnostic memory content is equivalent across the items and less than perfect (i.e., $A_{d}=B_{d}<1$ ), then whenever the level of nondiagnostic content for items from the A source is relatively greater than the level for the B source items, source accuracy under the "Source A?" 
cue frame will tend to be greater than source accuracy under the "Source B?" cue frame. This is because, as $A_{\text {nd }}$ and $\mathrm{B}_{\mathrm{nd}}$ diverge, the hit and false alarm rates in Equations 1 and 2 will also diverge as $\left(1-A_{d}\right) A_{n d}$ grows larger and $\left(1-B_{d}\right) B_{\text {nd }}$ grows smaller. Conversely, the same imbalance will lead the hit and false alarm rates in Equations 3 and 4 to converge as $\left(1-B_{d}\right) B_{n d}$ and $\left(1-A_{d}\right) A_{n d}$ play opposite roles; that is, the former expression now contributes to a decreasing hit rate and the latter to an increasing false alarm rate. Thus, on average, the model predicts that systematic differences in nondiagnostic item memory will lead to systematic differences in the estimate of accuracy under the two different cue frames (hits - false alarms). ${ }^{2}$

We refer to this as the IMM model relative accuracy prediction. It should be noted that this prediction is unaffected by whether or not subjects shift the overall "yes" response criterion between the two framings of the cue-for example, by becoming generally more likely to respond "yes" under one cue frame versus the other. This is because although such a systematic shift would generally elevate or depress the "yes" rates under one versus the other cue frame, the difference between correct and incorrect "yes" rates within each framing condition (viz., the accuracy) would remain largely unaffected by this general tendency, since it is this difference that determines the accuracy effect. A second important point about the model is that no commitment to the scaling of diagnostic and nondiagnostic memory evidence is needed in order for the predictions to hold. One can assume that both types of evidence are scaled continuously and are subject to a criterion, or that both reflect discrete retrieval states, or some combination of the two assumptions; regardless, the framing accuracy prediction still holds. ${ }^{3}$

In order to initially simulate the IMM model, we used Excel and randomly selected probability values from a uniform distribution for all four memory values $\left(A_{d}, B_{d}\right.$, $A_{n d}$, and $\left.B_{n d}\right)$ for 1,000 cases. Although dependencies between diagnostic and nondiagnostic retrieval are easily simulated, for brevity we only present simulation results in which all four values were sampled fully independently. However, the main conclusions were unaffected when the diagnostic information and nondiagnostic information for each type of item were instead allowed to become correlated. By extension, this suggests that the conclusions drawn from the simulation will be largely the same, regardless of whether or not one holds that source and item memory are correlated. The four sampled values were then plugged into Equations 1-4 to generate the correct and incorrect response rates under the two cue frames ("Source A?" and "Source B?"). Finally, two key values were calculated and compared. The first value, the relative source accuracy, was the difference in source accuracy across the two cue frame conditions [(Equation $1-$ Equation 2) - (Equation 3 - Equation 4)]. This merely represents the difference between correct and incorrect responding to the same types of items under the two different source cue frames. If the value is positive, accuracy is greater under the "Source A?" cue frame. If the value is negative, accuracy is greater under the "Source B?" cue frame. The second value calculated was based on an esti- mate of simple item recognition differences for the items. During simple recognition (e.g., "Have I seen this item before?"), all recovered item information should now become diagnostic. That is, even information that would have been insufficient for discriminating a specific source attribute is nonetheless perfectly useful in identifying the item as old. Given this, simple recognition should be a joint function of the availability of diagnostic and nondiagnostic information specified in the equations above. For A items, simple recognition is assumed to be monotonically related to Equation 1, and for B items, it is assumed to be monotonically related to Equation 3 . The key value computed for the simulation is the difference between these two estimates of recognition (Equation 1 - Equation 3). ${ }^{4}$ If this value is positive, the $\mathrm{A}$ items will yield greater recognition than $\mathrm{B}$ items, and vice versa if the value is negative.

Because on average both diagnostic and nondiagnostic item information should positively contribute to simple item recognition, the IMM model makes the unique prediction that differences in simple recognition for the two item types will themselves predict the cue-framing accuracy effect. This is because simple recognition differences should often suggest differences in the levels of nondiagnostic item memory across the two sources. More specifically, the model predicts that if simple recognition of A items exceeds recognition of B items, accuracy under the "Source A?" cue frame will on average exceed accuracy under the "Source B?" cue frame (the IMM relative accuracy prediction), and this prediction is possible even if the actual underlying levels of diagnostic and nondiagnostic content are unknown.

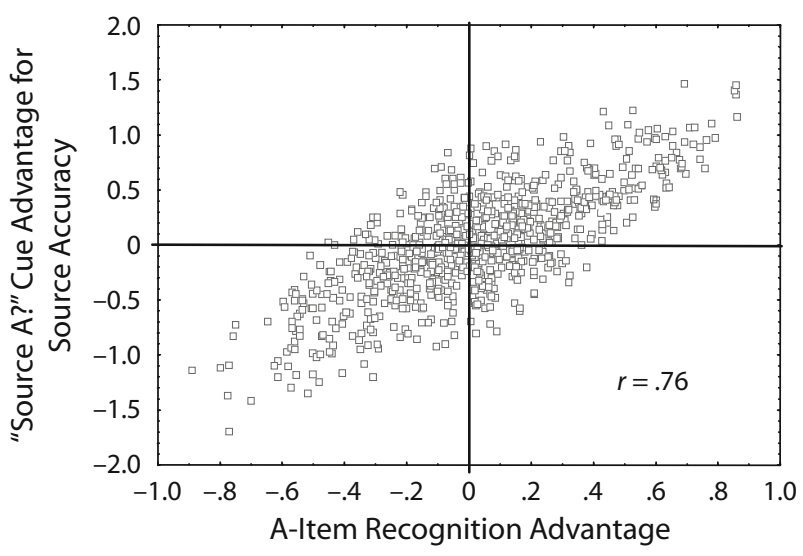

Figure 2. Outcome of the item memory misattribution model simulation. The $x$-axis indicates the difference in simple recognition scores for each case, with positive values indicating that recognition of the $A$ items was higher than recognition of the $B$ items (i.e., $A-B>0$ ). The $y$-axis indicates the difference in accuracy for the two source cue frames. Positive values indicate that the "Source A?" frame accuracy was higher, whereas negative values indicate that the "Source B?" frame accuracy was higher. The $r$ value is the correlation between the recognition difference and the cue-framing difference. A total of 1,000 cases were sampled, with the underlying values of diagnostic and nondiagnostic memory drawn randomly from uniform distributions. See the text and Equations 1-4 for information on calculation of the values. 
Figure 2 shows the relationship between the recognition difference and the IMM relative accuracy calculation from the simulation. In general, it is clear that the simulation confirms the qualitative predictions above. Differences in simple item recognition predict differences in the relative accuracy of source judgments under the two cue frames. More specifically, when the source cue frame uniquely specified the source with the higher-recognition items, accuracy was higher for that framing in $73 \%$ of the cases. In contrast, in only $27 \%$ of the cases did the framing highlighting the lower-recognition source yield higher accuracy. Finally, the value of the recognition difference correlated with that of the source accuracy difference $(r=.76)$.

Figure 3A plots the relationship between the recognition differences and cue-framing relative accuracy differences for cases in which the recognition of A items exceeded that of B items (the right half of the plot in Fig- ure 2). Only half of the data are displayed, since the model predictions are symmetric. When the data are plotted to show the probability of observing an accuracy difference favoring the "Source A?" cue frame, given a recognition difference favoring Source A items, one quantitative prediction of the model is easier to appreciate. Namely, as the recognition advantage of $A$ items relative to $B$ items grows increasingly large ( $x$-axis), a net source memory accuracy advantage for the "Source A?" relative to the "Source B?" cue frame becomes increasingly likely ( $y$-axis). For example, a recognition difference of less than $10 \%$ yielded no reliable prediction for relative accuracy, because half of the sampled cases yielded a higher source accuracy value for the "Source A?" frame, whereas the other half showed a "Source B?" frame advantage (leftmost bar). In contrast, when the A-item recognition advantage increased to between $10 \%$ and $20 \%$, approximately $70 \%$ of the cases

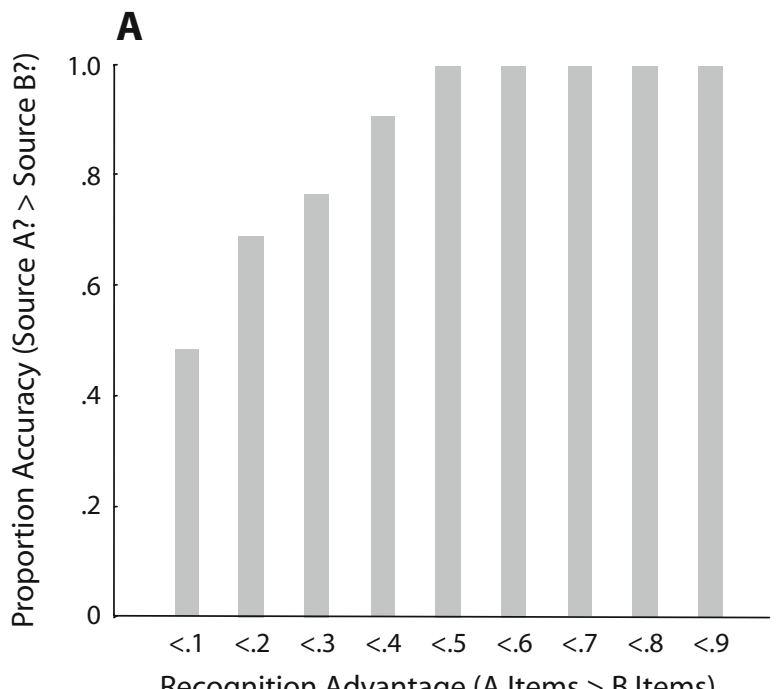

Recognition Advantage (A Items $>$ B Items)
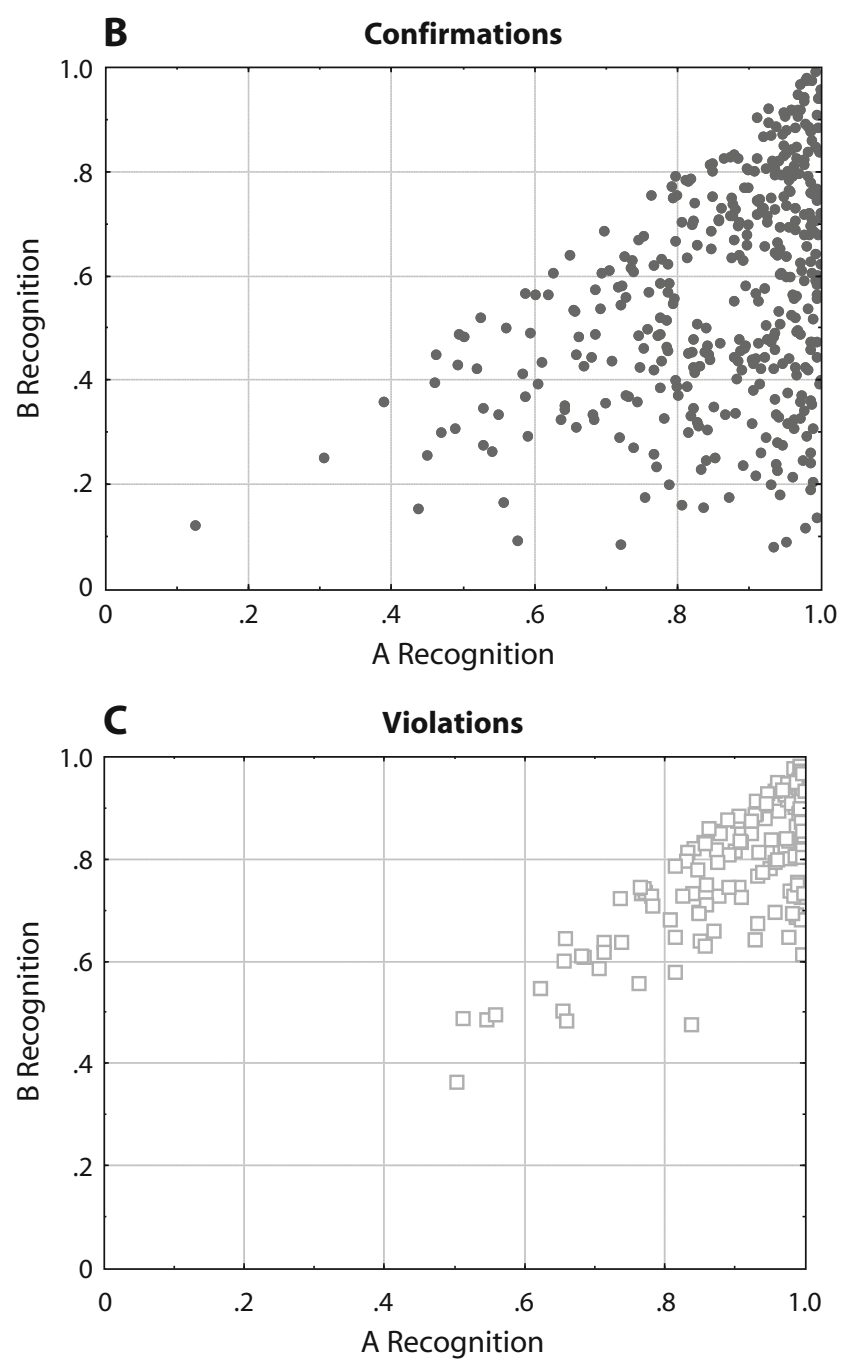

Figure 3. (A) Histogram simplifying the IMM model simulation predictions. For simplicity, data were restricted to cases in which recognition of $A$ items exceeded that of $B$ items. Each $x$-axis value indicates the size of the relative recognition difference, in steps of .1. For example, the second value bins cases in which the difference exceeded .1 but was less than .2 . The $y$-axis reflects the proportion of cases within each bin in which the relative source accuracy was in the same direction as the relative recognition score. That is, it shows the proportion of cases in which the accuracy under the "Source A?" cue frame exceeded accuracy under the "Source B?" cue frame. (B and C) Recognition values associated with cases in which the relative source accuracy score confirmed or violated the prediction. 
showed a source accuracy advantage for the "Source A?" over the "Source B?" frame. This continued as the recognition difference increased and reached ceiling when the difference exceeded $40 \%$, at which point all of the cases yielded a source accuracy advantage for the "Source A?" frame.

Finally, it is important to investigate the characteristics of the cases that failed to confirm the prediction. For example, the third bar of Figure 3A (.2 to .3) suggests that a little less than $80 \%$ of the cases in which recognition of $\mathrm{A}$ items exceeded that of B items by $20 \%-30 \%$ confirmed the prediction, yielding a source recognition advantage favoring the "Source A?" cue frame. What of the other 20\% of cases, which failed to support this prediction - are they systematically different in terms of recognition values? ${ }^{5}$ Figures $3 \mathrm{~B}$ and $3 \mathrm{C}$ show our initial investigation of this question by isolating the confirmations and violations of the prediction to see whether the absolute recognition values are informative. The points in both plots fall below the $\mathrm{A}$ recognition $=\mathrm{B}$ recognition diagonal because $\mathrm{A}$ items have greater recognition than B items. What is clear from the plots is that, in contrast to the majority of cases confirming of the prediction, violations of the prediction tend to occur whenever the recognition values are highly similar and/or when the recognition rates approach ceiling, with a large proportion of these violations clustered in the upper right-hand corner. Thus, the model suggests that the reliability of framing effects depends both on the relative difference in recognition between the source items and on the absolute level of recognition of the items, with extreme recognition values increasing the chances for violations. To investigate this idea further, we increased the number of cases in the simulation to 30,000 and isolated the data to the first four bins of recognition difference scores shown in Figure 3A. The goal was to see whether we could discover a clear pattern linking absolute recognition levels to the reliability of the cue-framing accuracy prediction. For example, if the recognition difference for a sample is between $20 \%$ and $30 \%$, does the level of absolute recognition also moderate the accuracy prediction?

Figure 4 indicates that the absolute recognition level does moderate the cue-framing effect predictions. In Figure 4, positive $y$ values indicate confirmation of the prediction, and negative values indicate violations. Consistent with the first bar in Figure 3A, Figure 4A shows that at a low recognition difference $(<10 \%)$, no framing effect is expected, regardless of the level of absolute recognition of the A items. However, this situation changes as the size of the recognition difference increases. For example, in Figure 4B the recognition difference is between $10 \%$ and $20 \%$, and consistent with Figure 3A, the majority of cases now confirm the prediction ( $\sim 70 \%)$, yielding positive $y$ values. However, observing a net positive accuracy effect in a sample of data now clearly depends on the absolute level of recognition, becoming less likely as recognition of the A items approaches ceiling. This can be further appreciated by following the linear fit indicated by the dotted line. Despite the fact that the data are clearly not bivariate normal, the fit line shows that the expected mean of a sample drawn from the population becomes increasing small, approaching zero, as the recogni- tion level increases. Furthermore, the variance in any given sample will also increase as the recognition of A approaches ceiling, further reducing the likelihood of observing a net effect in the sample. This pattern continues in panel C, although by the time one reaches a $30 \%-40 \%$ difference in recognition between A and B items (panel D), the effect would likely be robust, regardless of the absolute recognition level of A items. In general, Figure 4 demonstrates that for a given level of recognition difference, as the absolute recognition level increases, the mean of the framing effect values declines while the variance of these values increases. Thus, at low to moderate levels of recognition differences, observing reliable cue-framing effects is contingent on not having recognition rates approach ceiling. As the recognition difference increases, the effect of absolute recognition levels on the framing effect values becomes negligible.

On the basis of the equations and the simulation, the IMM model makes the following predictions:

Systematic differences in item recognition will lead to systematic cue-framing accuracy effects, such that the source containing the more easily recognized items will also demonstrate the greatest accuracy when it is solely specified in the cue frame. The strength of the prediction increases as the difference in recognition rates becomes prominent (Figure 3 ) and as the individual recognition rates move away from ceiling (e.g., in Figure 4).

Because the IMM model assumes that cue-framing effects reflect differences in item memory, it predicts that these effects should arise following a large array of encoding manipulations, as long as the manipulations result in uneven item recognition across queried sources. That is, the model assumptions suggest that this effect should be quite general.

We tested the IMM predictions across three experiments by manipulating the level of item recognition for probes drawn from the two queried sources and by examining different types of sources. Repetition manipulations were used to change item recognition levels, under the prediction that minimizing recognition differences or approaching ceiling recognition rates would eliminate the effect. In contrast, concrete and alphabetic order judgments were examined in order to see whether the cue-framing effect generalized beyond the read and generate tasks originally used by Marsh and Hicks (1998). Since concreteness and alphabetic judgments are assumed to produce clearly different levels of item memory (Craik \& Lockhart, 1972), the IMM model predicts a cue-framing effect for these sources as well.

\section{EXPERIMENT 1}

\section{Frequency Manipulation Generate/Read Tasks}

\section{Rationale}

This design parallels that of Marsh and Hicks (1998), but differs in two key respects. First, a standard recognition test was administered to gauge differences in item memory for conditions that would later be used during source memory testing (i.e., generated and read items). Second, a frequency manipulation was added during the study so that the trends predicted by the IMM model simulation could be tested. 
A $\quad 0 \%-10 \%$ Recognition Difference (A>B)

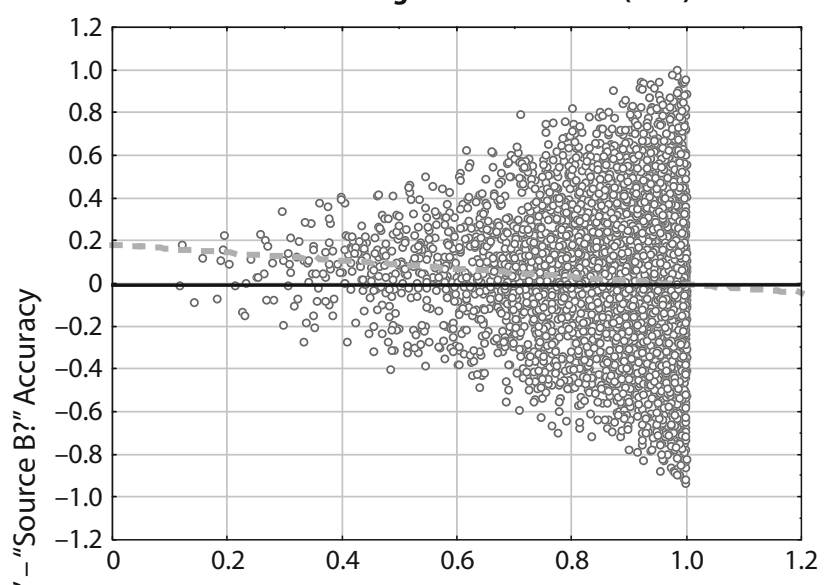

C $20 \%-30 \%$ Recognition Difference $(A>B)$

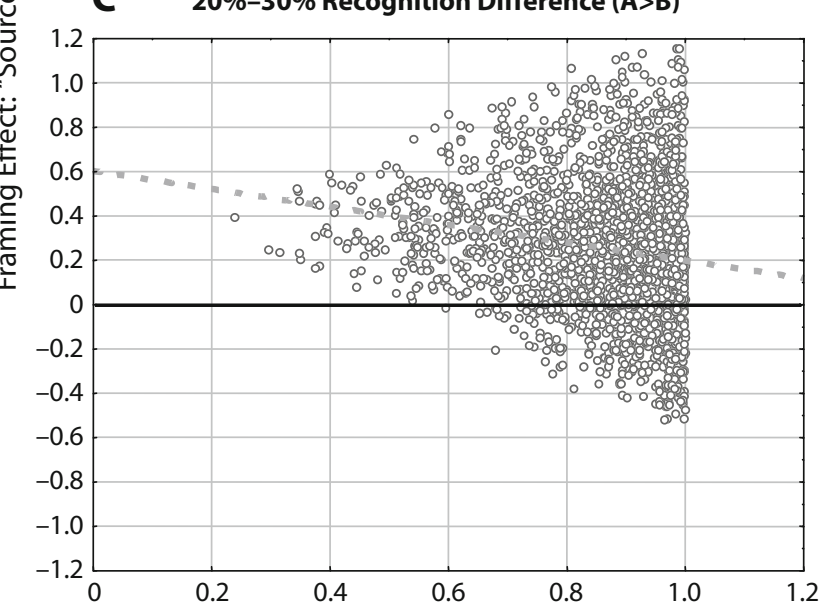

B

10\%-20\% Recognition Difference (A>B)

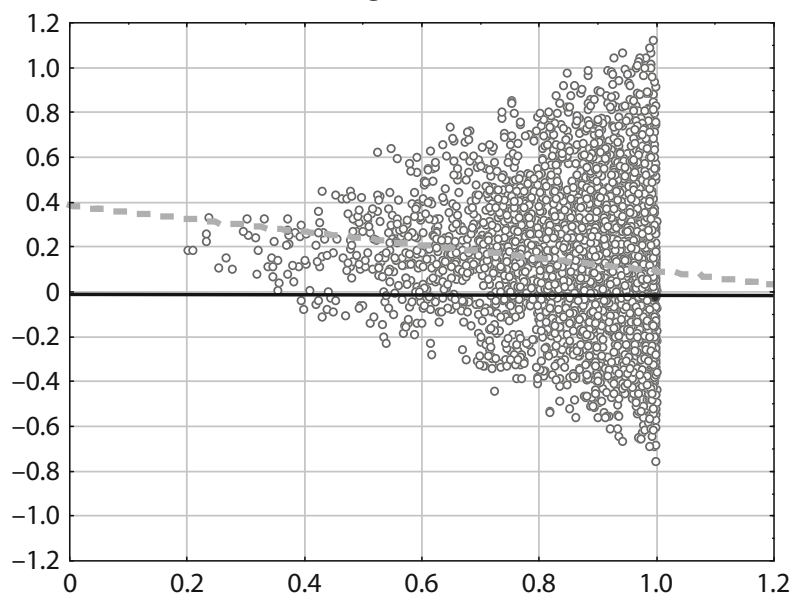

D 30\%-40\% Recognition Difference (A>B)

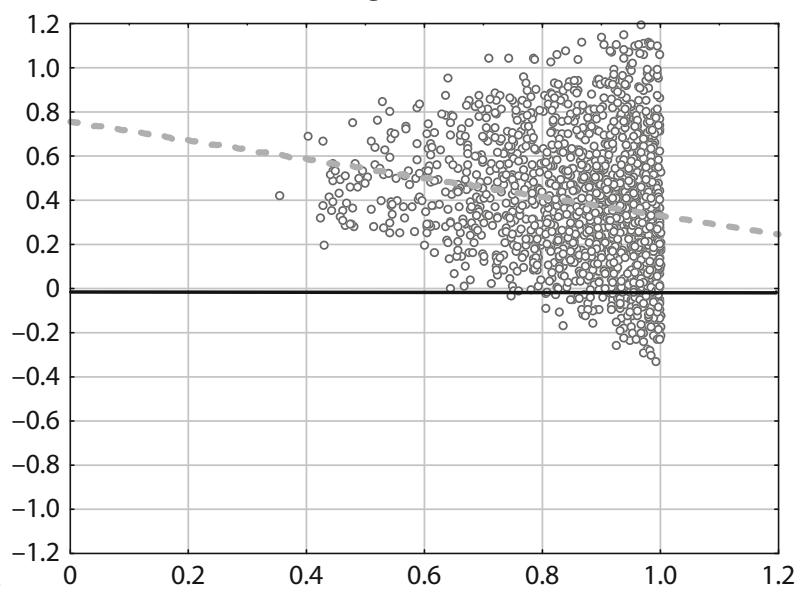

A-Item Recognition Level

Figure 4. Scatterplots illustrating the link between the reliability of the cue-framing effect and the absolute recognition level of $A$ items. The $y$-axes indicate the relative source accuracy score, with positive values indicating that the score favored the "Source A?" frame as highlighting the items with the greatest recognition. The $x$-axes indicate the recognition value of the $A$ items. The dark horizontal bar is the zero-difference line, which shows that as the relative recognition difference increases, a greater proportion of the cases confirm the prediction (viz., they appear above the line). The dashed line is the simple linear fit of the relation between the A-item recognition level and the mean relative accuracy score. This line illustrates that as $A$-item recognition increases, the mean of the relative accuracy scores decreases. Furthermore, as A-item recognition increases, the variance of the accuracy scores increases. Both effects indicate that increasing values of $A$-item recognition make positive cue-framing accuracy effects increasingly unlikely.

\section{Method}

As in Marsh and Hicks (1998), the two encoding sources were created by having subjects either generate items from anagrams or simply read the words intact.

Subjects. Fourteen volunteers participated in return for a payment of $\$ 10$. Testing was conducted in accordance with Duke University regulations for protecting human subjects, and the session lasted approximately $45 \mathrm{~min}$.

Materials and Procedure. For each subject, three lists of 120 medium-frequency words were drawn from a pool of 1,075 items. For each list, 30 items were randomly assigned to be solved as anagrams, and 30 other items to be seen intact during encoding. The remaining 60 items were used as lures during testing. Of the 30 words chosen to be solved as anagrams or seen intact at encoding, 10 were studied either once, twice, or three times. This repetition was predicted to increase some combination of both diagnostic and nondiagnostic item memory for the materials. For the source-monitoring and recognition tests, all 120 items (generated, seen, and new) were randomized anew and presented individually, with the only difference across tests being the nature of the retrieval cue.

During presentation of each 60 -item encoding list, the nature of the task was determined by the font format of the presented string. Words presented in all lowercase (e.g., "pickle") were simply to be read silently. However, if the word contained two capital letters, it was an anagram to be solved (e.g., "pLckIe"). Subjects were informed that the second and next-to-last letters were to be mentally swapped in order to solve the anagram. Responding was self-paced, and subjects proceeded by pressing the space bar for each item. However, to ensure similar viewing times for both read items and anagrams (which rarely required more than $3 \mathrm{sec}$ to solve), all items remained on screen for a minimum of $3 \mathrm{sec}$.

The only manipulation across the three tests was the nature of the retrieval cue. The first test for all subjects was simple old/new recognition with confidence ratings. Each subject was instructed that the test list would contain both items seen during the immediately preceding study tasks and new items. Subjects were instructed to 
indicate the old/new status $(1=y e s, 2=n o)$ and to give their confidence in their answer $(1=$ certain, $2=$ moderate, $3=$ guessing $)$. Responding was self-paced.

The remaining two tests were source memory tests in which the cue prompted endorsement of either previously solved anagrams $(1=$ yes, $2=n o)$ or previously read items $(1=$ yes, $2=$ no $)$. Items from the nontarget source and new items were to be assigned a "no" response. As with the recognition test, each response was followed by a confidence rating. All words at test were presented intact and in lowercase font. The order of the latter two test types/cue frames ("Generated?" or "Read?") was randomized across subjects, such that 6 of them received the "Generated?" format first, whereas 8 received the "Read?" format first.

\section{Results}

Recognition performance: Test 1 . The false alarm rate to new items in this mixed list was .13. The hit rates for the recognition test are shown in Table 1, broken down by encoding frequency and encoding task. In the case of items seen once, which approximates the encoding conditions in Marsh and Hicks (1998), there is a large recognition advantage for the generated versus the read items [.77 vs. .50; $t(13)=3.45, p<.01]$, and neither recognition value approaches ceiling levels. For items seen twice, the difference, though numerically smaller, was also significant $[.96$ vs. $.75 ; t(13)=4.00, p<.01]$, and the difference remained significant for items seen three times [.96 vs. $.86 ; t(13)=2.38, p<.05]$, although this was the smallest difference in absolute terms. In the latter two cases, cueframing effects were predicted to be unlikely. For items seen three times, the recognition difference is too small to yield a reliable effect (see Figures $3 \mathrm{~A}$ and $4 \mathrm{~A}$ ). In contrast, for twice-repeated items, the recognition difference is .21, but the absolute recognition level for generated items is .96. Looking at either panel B or C of Figure 4 suggests that a net cue-framing effect, if observed, would be very small and unreliable with an absolute recognition rate this high. There is also a case in which the item recognition hit rates do not appear to differ across the encoding tasks. More specifically, generated items seen once were endorsed at a rate similar to that of read items seen twice $[.77$ vs. . $75 ; t(13)<1]$. Because these values are so similar, in-
Table 1

Experiment 1 Recognition Performance

\begin{tabular}{|c|c|c|c|c|c|c|c|c|}
\hline \multirow[b]{3}{*}{ Origin } & \multicolumn{6}{|c|}{ Frequency } & & \\
\hline & \multicolumn{2}{|c|}{ Once } & \multicolumn{2}{|c|}{ Twice } & \multicolumn{2}{|c|}{ Thrice } & \multicolumn{2}{|c|}{ Matched } \\
\hline & $\bar{M}$ & $S D$ & $\bar{M}$ & $S D$ & $\bar{M}$ & $S D$ & $\bar{M}$ & $S D$ \\
\hline Generated & .77 & .14 & .96 & .08 & .96 & .07 & & \\
\hline Read & .50 & .27 & .75 & .22 & .86 & .18 & & \\
\hline Difference & .27 & .30 & .21 & .19 & .11 & .21 & .02 & .21 \\
\hline
\end{tabular}

Note-Mean "yes" rates during item recognition as a function of encoding frequency and task. Difference indicates the difference in hit rates for generated versus read items. The Matched value is the difference in hit rates for generated and read items considered matched in terms of recognition; these rates are highlighted in bold. The false alarm rate for new items was .13.

dicating no appreciable recognition difference, the model again predicts that there should not be a systematic IMM effect if these two item types were used in the equations. Finally, for once-seen items, the recognition difference is fairly prominent at .27 , and the average recognition level of .77 is well below ceiling; thus, a prominent cue-framing effect should be observed favoring the "Generated?" cue frame (see Figure 4C).

Overall, in light of these recognition data, the IMM model predicts that the relative accuracy effects of the cue frame should be most evident for items seen once. In the remaining conditions, they should be small and unreliable, because as repetitions increased, the absolute recognition scores approached ceiling and/or the relative difference between the recognition rates declined sizably.

Source performance: Tests 2 and 3. Table 2 shows the endorsement rates for the two cue frames as a function of encoding frequency and source origin. To test for the presence of a cue-framing effect, we simply computed the difference in relative accuracy under the cue frames in the same manner as in the simulation. More specifically, for each subject we computed the difference between correct and incorrect "yes" rates under the "Generated?" cue frame, minus the difference between correct and incorrect response rates under the "Read?" cue frame [i.e., (Equation 1 - Equation 2) - (Equation 3 - Equation 4)

Table 2

Experiment 1 Source Memory Performance

\begin{tabular}{|c|c|c|c|c|c|c|c|c|c|}
\hline \multirow[b]{3}{*}{ Cue Frame } & \multirow[b]{3}{*}{ Origin } & \multicolumn{6}{|c|}{ Frequency } & & \\
\hline & & \multicolumn{2}{|c|}{ Once } & \multicolumn{2}{|c|}{ Twice } & \multicolumn{2}{|c|}{ Thrice } & \multicolumn{2}{|c|}{ Matched } \\
\hline & & $\bar{M}$ & $S D$ & $M$ & $S D$ & $\bar{M}$ & $\overline{S D}$ & $M$ & $S D$ \\
\hline \multirow[t]{2}{*}{ "Generated?" } & Generated & .61 & .16 & .78 & .13 & .86 & .18 & & \\
\hline & Read & .23 & .24 & .26 & .31 & .21 & .29 & & \\
\hline \multirow{2}{*}{ "Read?" } & Read & .56 & .19 & .81 & .15 & .85 & .17 & & \\
\hline & Generated & .36 & .22 & .25 & .27 & .20 & .24 & & \\
\hline Relative accuracy & & .17 & .30 & -.04 & .41 & 00 & .53 & -.11 & .39 \\
\hline
\end{tabular}

Note-Mean "yes" rates during source memory. Cue Frame indicates the cue presented at test, whereas Origin indicates whether items were drawn from a prior generate or read task. The vertical ordering of origins corresponds to correct, then incorrect, responding under each frame. Frequency indicates the number of encoding repetitions. Cells in bold are assumed to be equated in terms of item recognition, as determined by the recognition performance data shown in Table 1. Relative accuracy indicates the difference in source accuracy for the "Generated?" minus the "Read?" cue frame. The Matched value column is the accuracy calculation for items generated once versus read twice, which are assumed to be matched in terms of recognition accuracy and are highlighted in bold [i.e., $(.61-.26)-$ $(.81-.36)=-.11$, based on rounded values]. 
above]. If this difference reliably falls above zero, the subjects are more accurate under the "Generated?" than under the "Read?" cue frame, with the former highlighting the items known to have greater recognition. For example, looking at Table 2, in order to calculate the cue-framing accuracy effect for the once-presented items, one uses the following equation: $(.61-.23)-(.56-.36)$. For the three frequency conditions, this value only significantly differed from zero for the seen-once condition $[t(13)=$ $2.18, p<.05]$. There was no evidence of a cue-framing effect under the two- and three-presentation conditions $(t \mathrm{~s}<1)$. Because simple recognition of once-generated and twice-read stimuli was matched (see the recognition analysis above and Table 2), we also compared the framing relative accuracy effect for these items. This comparison was also not significant $[t(13)=-1.01, p>.33]$. Finally, the intrusion rates for entirely new items were affected by the source cue framing, with intrusions occurring more often for the "Read?" than for the "Generated?" cue frames [.33 vs. .14; $t(13)=3.12, p<.01]$. This might indicate a shift in the "yes" response criterion across the cue frames, but as discussed above, this does not affect the cue-framing accuracy predictions.

In general, the source accuracy data support the IMM model. Cue-framing accuracy effects favoring the "Generated?" cue required prominent differences in relative recognition across generated and read items and an absence of absolute recognition ceiling effects. As the relative recognition difference declined and/or recognition rates approached ceiling, the cue-framing accuracy effect disappeared. Furthermore, when items were matched in terms of recognition, the cue-framing effect was also eliminated.

\section{EXPERIMENT 2 Alternate Source Tasks}

\section{Rationale}

If the IMM model is correct, cue-framing accuracy effects should be relatively commonplace, occurring often when systematic and prominent item recognition differences exist across queried sources. To test the generality of such effects, we chose two tasks that should clearly lead to different recognition levels, yet do not easily fit into a perceptual versus cognitive monitoring distinction (or into an internal vs. external distinction) potentially applicable to the tasks in Experiment 1. The chosen tasks were semantic ratings of word concreteness and alphabetic order judgments of the first and last letters of presented words. Although an alphabetic judgment is no more perceptual or external in nature than a semantic judgment, the IMM clearly predicts that cue-framing accuracy effects will occur, because item memory levels should differ substantially as a function of levels of processing.

\section{Method}

Subjects. Sixteen volunteers participated in return for course credit. Testing was conducted in accordance with Duke University regulations for protecting human subjects, and the session lasted approximately $1 \mathrm{~h}$. Data from 1 subject were excluded due to nearchance performance.
Materials and Procedure. The procedure consisted of four study/test cycles, with each cycle composed of a study section followed immediately by source memory testing. During study, half of the items were encoded using an alphabetic rating task and the other half using a concrete/abstract task; the items were randomized on a trial-by-trial basis, with 80 items presented during each study phase (40 for each task). Words were presented singly underneath a cue designating the encoding task, either "In alphabetical order? $1=$ yes $2=$ no" or "Concrete? 1 = yes $2=$ no." For the alphabetical order task, subjects were instructed to determine whether the first and last letters of the word were in alphabetical order. For the concrete/ abstract task, subjects decided whether each word referred to a concrete physical object or an abstract concept. Responding was selfpaced, and the perceptual forms of the words were identical across the encoding tasks.

During test, these same items were re-presented in the same order, resulting in a constant study/test lag, and source memory was queried using one of two cue frames throughout each entire test block. For half of the tests, the cue frame read "Concrete/abstract task? $1=$ yes 2 = no," whereas for the other half it read "Alphabetical task? 1 = yes 2 = no." The order of the cue frame manipulation across blocks was randomized for each subject, and there were no new items present in the test lists. Subjects were required to respond within $2 \mathrm{sec}$, and they were prompted to respond more quickly if they failed to do so. Following each response to the source memory cue, a self-paced confidence report was collected.

On-screen instructions were displayed before each study and test section, with an opportunity for subjects to ask questions if necessary.

\section{Results and Discussion}

Consistent with the levels of processing difference, these two encoding tasks yielded prominent recognition differences during a simple recognition paradigm conducted using different subjects. Consistent with the IMM model, the data from source memory testing also demonstrated a clear accuracy advantage for the "Concrete?" relative to the "Alphabetic?" cue frame $[t(14)=3.27, p<.01]$. That is, the encoding task that yielded greater item recognition also yielded the greatest source accuracy when specifically framed in the source retrieval cue (Table 3). Under the model, this indicates that the items previously rated for concreteness yielded greater levels of nondiagnostic item memory content than did the items arising from the alphabetic sorting task.

\section{EXPERIMENT 3 Alternate Frequency Manipulation}

\section{Rationale}

Experiments 1 and 2 suggested that the cue-framing accuracy effect was systematically related to two aspects

Table 3

Source Memory Performance for Experiment 2

\begin{tabular}{|c|c|c|c|c|}
\hline \multirow[b]{3}{*}{ Item Origin } & \multicolumn{4}{|c|}{ Cue Frame } \\
\hline & \multicolumn{2}{|c|}{ Concrete? } & \multicolumn{2}{|c|}{ Alphabetic? } \\
\hline & $M$ & $S D$ & $M$ & $S D$ \\
\hline Concrete task & .85 & .04 & .27 & .19 \\
\hline Alphabetic task & .25 & .11 & .60 & .20 \\
\hline Relative accuracy & .26 & .31 & & \\
\hline
\end{tabular}

Note-Means are "yes" rates during the source memory task. Relative accuracy denotes the difference in accuracy measures for the "Concrete?" frame minus the "Alphabetic?" frame. 
of recognition performance, the relative recognition difference and the absolute level of recognition. When recognition differences were prominent and recognition scores well away from ceiling, the effect was likely to be observed. Conversely, if one minimized recognition differences between the sources and/or increased the recognition rates toward ceiling, a reliable effect became increasingly unlikely. Experiment 1 used item repetition during study to minimize or eliminate item recognition differences and drive recognition rates to ceiling. However, repeating items using the same source tasks that are later queried may be less than ideal, since it is possible that this would not only increase recognition but simultaneously also boost source retrieval enough to overcome hypothetically inefficient monitoring strategies. From this perspective, one could argue, for example, that the "Read?" cue frame during Experiment 1 still led subjects to adopt a less effective perceptual monitoring strategy, but that this deleterious effect was offset by the increased ability to recover diagnostic perceptual information because of the repeated encoding. To rule out such an interpretation, it is necessary to increase the nondiagnostic content of the items independently of the source tasks later queried. Experiment 3 did this by using a different repetition method: Instead of repeating items using the same tasks that are later queried during the source memory test, we repeated them using a third, orthogonal task that was never queried during later testing. Even if subjects were able to explicitly recover specific information about this tertiary task, it would clearly be unrelated to the sources being queried during the test. Nonetheless, the IMM model predicts that subjects will be influenced by this tertiary task because it will boost item recognition, and this constitutes a strong test of the model's assumption that subjects use nondiagnostic item memory during confirmatory source attributions.

\section{Method}

Subjects. Nineteen volunteers participated in return for course credit. Testing was conducted in accordance with Duke University regulations for protecting human subjects, and the session lasted approximately $1 \mathrm{~h}$. Data from 3 subjects were excluded due to poor performance or programming error.

Materials and Procedure. The procedure was similar to that in Experiment 2 and consisted of four study/test blocks. Two of the test blocks involved recognition testing, and two involved source memory testing. The study sections of all blocks were identical, and the order of source and item recognition blocks was randomly determined for each subject. For each study/test run, a list of 120 items was randomly selected from the same word pool as in Experiment 2. In Study Phase 1, 80 of these items were studied, with half assigned to the alphabetic judgment and half to the concreteness judgment. Following this first phase, half of the items from each prior encoding task were then assigned to Study Phase 2, during which subjects made syllable-counting judgments ("Two or more syllables? $1=$ yes, $2=$ no") that were repeated three times for each item in a randomized order. The main purpose of this Phase 2 manipulation was to increase the item recognition for the items, under the assumption that this would influence the later source attributions.

Thus, at the completion of Study Phase 2, there were four item conditions: (1) deeply processed (concreteness) items seen once during the initial phase, (2) shallowly processed (alphabetic) items seen once during the initial phase, (3) deep items seen once during the initial phase and then three times during the syllable-counting phase, and (4) shallow items seen once during the initial phase and then three times during the syllable-counting phase. These items were then tested in either a source memory or item recognition test immediately following Study Phase 2. For the source memory test, the 80 items were randomized, with half assigned to one cue frame ("Concrete/abstract task? 1 = yes $2=$ no") and the other half to the alternate cue frame ("Alphabetical task? 1 = yes $2=$ no"). Thus, there were eight total conditions with 10 probes each. No new items were presented during source testing, so only 80 of the original 120 selected list items were used. The instructions presented prior to source testing made no mention of the syllable-counting task during Phase 2 and focused entirely on the two initial (Study Phase 1) source origins that would be specifically queried during the test. In contrast, during item recognition, the 80 studied items were represented along with the remaining 40 unstudied items of the original list. The order of items was randomized with respect to the study order, and half of the items received one version of an old/new cue frame ("Old item? 1 = yes $2=$ no") and half the complementary version ("New item? 1 = yes $2=$ no"). Because the recognition results were unaffected by the form of the recognition cue, we only report the data for the "Old item?" cue variant below. Responding was self-paced throughout.

\section{Results and Discussion}

Recognition blocks. Unsurprisingly, for items seen only once, a within-subjects $t$ test demonstrated a large advantage in hit rates for the deeply processed over the shallowly processed items $[.86$ vs. . $60 ; t(15)=10.03, p<$ .001 ; see Table 4]. When items from these two tasks were further repeated in the syllable-counting task (three additional repetitions), the advantage for the concreteness task items was significantly reduced [.25 vs. . $11 ; t(15)=$ $4.27, p<.001]$, although it remained reliable [.96 vs. .85; $t(15)=3.15, p<.01]$. Critically, however, the repetition clearly pushed recognition toward ceiling for the deep task items and reduced the recognition difference across the two types of items. Finally, when the concrete task items seen once were compared with the alphabetic task items that received additional repetition during Phase 2 (bold recognition rates in Table 4), there was no discernible difference in item recognition $[.86$ vs. $.85 ; t(15)<1]$. Thus, these two conditions can be considered well matched in terms of item recognition levels.

Overall, the recognition data demonstrate an advantage in item recognition for the concrete versus the alphabetic task. This recognition advantage declined when the items were further repeated in a subsequent syllable-counting

Table 4

Recognition Results From Experiment 3

\begin{tabular}{|c|c|c|c|c|c|c|}
\hline \multirow[b]{3}{*}{ Origin } & \multicolumn{4}{|c|}{ Frequency } & & \\
\hline & \multicolumn{2}{|c|}{ Low } & \multicolumn{2}{|c|}{ High } & \multicolumn{2}{|c|}{ New } \\
\hline & $\bar{M}$ & $S D$ & $M$ & $S D$ & $\bar{M}$ & $S D$ \\
\hline Concrete task & .86 & .11 & .96 & .06 & .16 & .13 \\
\hline Alphabetic task & .60 & .15 & .85 & .15 & & \\
\hline Difference & .25 & .10 & .11 & .14 & & \\
\hline
\end{tabular}

Note-Means are proportions of "yes" responses as a function of encoding task and repetition frequency. Low-frequency items were seen once during the initial Phase 1 study condition. High-frequency items were seen once during Phase 1 and at three additional times during the Phase 2 study condition. Proportions in bold reflect similar recognition levels across the two tasks. 
task prior to testing, and the absolute rate for the deep items approached ceiling. On the basis of these patterns, and as in Experiment 1, the IMM model predicts cueframing effects for the items seen only once during the initial tasks. In contrast, this framing effect should decline or disappear for the repetition condition items, and should also be absent when restricting the analysis to alphabetic task items under repetition versus concreteness rating items seen only once, because these items have similar recognition levels.

Source memory blocks. Table 5 shows the endorsement rates for the two cue frames as a function of encoding frequency and source origin. For the low-frequency condition, there was a significant cue-framing accuracy effect favoring the "Concrete?" over the "Alphabetic?" cue frame $[t(15)=2.65, p<.05]$. This effect was numerically smaller and only approached significance for the high-frequency items $[t(15)=2.00, p>.064]$, and this failure was predicted, given the low relative recognition difference and the high absolute recognition scores in this condition (see Figure 4B). Finally, the effect was eliminated altogether when items were matched for recognition levels $[t(15)=1.16, p>.26]$.

One unanticipated finding was the failure of the highfrequency condition to yield more robust increases in "yes" rates than the low-frequency condition (Table 5). More specifically, accuracy for the concreteness items under the "Alphabetic task?" cue did not change across frequencies, and in the remainder of cells the increases were small, only approaching significance $(p s>.10)$. This could reflect a general tendency for observers to treat items remembered as repeated cautiously_-perhaps because they suspected that the repetition manipulation was a trick to elicit incorrect endorsements. This is not a problem for the test of the high-frequency condition, because all the values in the equations would be subject to the same criterion. However, this is not true for the matched analysis, because the values were taken from the low-frequency concrete items and the high-frequency alphabetic items (see bold values in Table 5), which recognition testing indicated were well matched for simple recognition. Looking at the IMM equations, it is clear that in the matched analysis a more conservative criterion for the high-frequency alphabetic items would serve to reduce their intrusion under the "Concrete task?" frame (Equation 2) and their detection under the "Alphabetic task?" frame (Equation 4), and this would have a net effect of artificially inflating the relative accuracy score for the matched analysis. However, this would mean that the relative accuracy for the matched condition in the absence of a criterion shift would have been even smaller than the obtained estimate of .04, which further argues against any reliable advantage for the "Concrete task?" frame.

One potential objection to the use of the tertiary task for increasing item recognition is that it may have somehow caused reminding of the initial source encoding conditions, essentially serving to provide additional encoding opportunities for the original sources. If this had occurred, the cue-framing effect might have been eliminated for the high-frequency condition simply because memory for the original sources was boosted enough to overcome any possible deleterious effects of inefficient monitoring strategies. We tested this alternative hypothesis by separately comparing source accuracy for the high- versus the lowfrequency items within each cue frame condition - for example, comparing the source accuracy under the "Concrete task?" frame for low-frequency items with that of the high-frequency items under the same framing. If reminding had occurred, source discrimination should then have been superior for the high-frequency items, since more encoding opportunities would have accrued. However, accuracy for the high-frequency items was not greater under either the "Concrete task?" $[t(15)<1]$ or the "Alphabetic task?" cue frame $[t(15)=1.18, p>.25]$, ruling out the reminding hypothesis.

Overall, the relative accuracy findings support the IMM model. Again, the cue frame highlighting the items with greater item recognition elicited higher source accuracy

Table 5

Source Memory Results From Experiment 3

\begin{tabular}{|c|c|c|c|c|c|c|c|}
\hline \multirow[b]{3}{*}{ Cue Frame } & \multirow[b]{3}{*}{ Origin } & \multicolumn{4}{|c|}{ Frequency } & \multirow{2}{*}{\multicolumn{2}{|c|}{ Matched }} \\
\hline & & \multicolumn{2}{|c|}{ Low } & \multicolumn{2}{|c|}{ High } & & \\
\hline & & $M$ & $\overline{S D}$ & $M$ & $S D$ & $M$ & $S D$ \\
\hline \multirow[t]{2}{*}{ "Concrete task?" } & Concreteness & .83 & .13 & .87 & .09 & & \\
\hline & Alphabetic & .34 & .19 & .40 & .19 & & \\
\hline \multirow{2}{*}{ "Alphabetic task?" } & Alphabetic & .59 & .13 & .67 & .18 & & \\
\hline & Concreteness & .27 & .21 & .27 & .17 & & \\
\hline Relative accuracy & & .17 & .26 & .08 & .15 & .04 & .13 \\
\hline
\end{tabular}

Note-Means are "yes" rates during source memory. Cue Frame indicates the cue presented at test, whereas Origin indicates whether an item was drawn from a prior concrete or alphabetic rating task. The vertical ordering of origins corresponds to correct, then incorrect, responding under each frame. Frequency indicates the number of encoding repetitions. Relative accuracy indicates the difference in source accuracy under the concrete task minus the alphabetic task cue frame. The Matched column is the accuracy calculation for items rated once for concreteness versus items rated for alphabetic order that were also repeated three times during a subsequent syllable-counting task. These conditions yielded comparable (i.e., "matched") simple recognition scores that are indicated in bold in the table. 
than did the frame that instead highlighted the items that yielded lower recognition. The magnitude of this relative accuracy advantage systematically declined as recognition differences also declined and as recognition levels approached ceiling (matched and high-frequency contrasts). Importantly, the effect was successfully eliminated even when the repetition manipulation was done via a tertiary task unrelated to the tasks targeted by the source memory cue frames. This result rules out the notion that eliminating the effect via repetition occurs because of gains in the pertinent source memories accrued through that repetition.

\section{GENERAL DISCUSSION}

Taken as whole, the three experiments provide considerable support for the IMM model. Under this model, cue-framing accuracy effects reflect the heuristic use of item memory information during confirmatory source decisions. The expression of the heuristic is contingent on the absence of diagnostic source retrieval and reflects systematic imbalances in item memory for items drawn from the two queried sources. Equations 1-4 formalize this relationship and assume that nondiagnostic information is used in a less controlled fashion than diagnostic content (cf. Jacoby, 1991). Importantly, even in the absence of knowledge about the specific levels of diagnostic and nondiagnostic information present, the Monte Carlo simulations predicted a clear relationship between relative item recognition differences, absolute item recognition levels, and the likelihood of observing the cue-framing accuracy effect. In general, the model predicts that the effect is increasingly likely to be observed as recognition differences between the two classes of source items increase, and as absolute recognition levels decrease. Conversely, as recognition differences decrease and/or recognition rates approach ceiling levels, the model predicts that the framing effect should become highly unreliable. Because the model does not assume different monitoring strategies, the prediction generally applies in situations leading to systematic recognition imbalances across the two queried source origins - for example, whenever levels of processing differ considerably. Without rehashing the specific results of Experiments 1-3, all of these model predictions were confirmed.

As noted in the introduction, there is a large body of research demonstrating that subjects are prone to misattributing item memory when recovery of specific diagnostic content is limited (e.g., Huppert \& Piercy, 1976, 1978; Jacoby, Kelley, et al., 1989; Jacoby, Woloshyn, \& Kelley, 1989; Kelley \& Wixted, 2001; Koriat \& Levy-Sadot, 2001; Lindsay et al., 1997; Whittlesea et al., 1990). Although the IMM model explicitly characterizes how item memory contributes to cue-framing effects, it does not explain why subjects use this item information when they ostensibly should have little reason to believe it is useful. We suggest that this occurs because in everyday contexts, this information is in fact pertinent for confirmatory source judgments. Similarly, although Marsh and Hicks (1998) did not conclude that item recognition drove the cue-framing accuracy effect, they did note a general increase in endorsement rates for the confirmatory cue frames used here versus the more traditional ones allowing three options on each trial (e.g., Source A, Source B, or new). Drawing a distinction between laboratory versus everyday source memory demands, they noted that in the everyday environment, subjects often try to confirm a particular source using questions such as "Did you read that in the newspaper?, Did you see that on the news?, Did Jennifer tell you that?" (p. 1149). As Marsh and Hicks suggested, this preference for naturally framing source problems in a confirmatory manner (e.g., Is this from source A?) probably reflects the fact that the number of alternate source candidates in everyday contexts is likely too large to be simultaneously or exhaustively considered. In addition, however, we believe that the preference also reflects the natural tendency of subjects to adopt a confirmatory stance during hypothesis testing under noisy or uncertain conditions (Nickerson, 1998). If subjects naturally frame source tasks in a confirmatory manner, then outside the laboratory, item memories consistent with the general description (either recollective or familiarity based) of the considered source are indeed relevant. Items that are perceived as familiar, and/ or that evoke remembrances consistent with the gist of the source description currently held in mind, are clearly more viable candidates than items that strike one as novel or fail to evoke any such remembrances. Thus, we suggest that the influence of item memory demonstrated in the present design, and similarly observed in a host of other studies, potentially reflects the fact that subjects naturally adopt a confirmatory stance when deciding about their memories. In short, we suggest that the tendency for subjects to heuristically use availability or similarity that has been demonstrated in numerous other decision-making contexts (for a review, see Gilovich et al., 2002) also operates during episodic memory attributions when retrieval cues are framed in a confirmatory manner.

\section{Combining the Strategic Monitoring and IMM Accounts}

In comparison with the strategic monitoring characterization, the IMM model provides a more general account of the present data, since it assumes the same mechanisms across all three experiments. Furthermore, the IMM model is the only one we are aware of that makes systematic predictions linking the reliability of the cue-framing effect to item recognition differences and overall item recognition levels. However, this is not meant to suggest that other operations, including strategic monitoring, do not also play a critical role in source memory outcomes, which is inarguably the case (Dobbins, Foley, Schacter, \& Wagner, 2002; Dodson \& Johnson, 1993; Johnson et al., 1993). Whereas the strategic monitoring account emphasizes the use of the qualitative characteristics of memory traces during source attribution, the IMM model simply complements this view by also considering what subjects do, if anything, with item memories related to the general gist of the prior encounter specified in the cue frame. In this sense, the IMM model can be seen as a complementary heuristic or simple attribution strategy that deals with recovered content not typically considered under the strategic monitoring account. 
Although the IMM predicted the observed relationships between cue-framing effects and item memory levels, it could well be the case that operations more in line with strategic monitoring are also in play. One way to incorporate both heuristics into the present model would be to assume that the recovery of diagnostic memory content for the deeply processed items (e.g., $A_{d}$ ) is in fact affected by active search or monitoring strategies induced by the cue frame, and that in the absence of diagnostic content recovery, subjects use nondiagnostic memory information (e.g., $\mathrm{A}_{\mathrm{nd}}$ ) in an uncontrolled or more heuristic fashion. This would essentially serve to amplify the effect captured in Equations 1-4, because reducing the diagnostic content across frames enables the nondiagnostic content differences to be expressed more fully. More specifically, if the diagnostic content of $\mathrm{A}$ and $\mathrm{B}$ items is initially equal and less than $1\left(\mathrm{~A}_{\mathrm{d}}=\mathrm{B}_{\mathrm{d}}<1\right)$, and the $\mathrm{A}$ items yield more nondiagnostic content than do the $\mathrm{B}$ items, then Equations 1-4 predict a cue-framing accuracy effect favoring the "Source A?" cue frame. Now, if one further assumes that, when transitioning from the "Source A?" to the "Source B?" cue frame, diagnostic content for the A items becomes less available because of controlled monitoring strategies, then the intrusion of $\mathrm{A}$ items will further increase as Equation $4\left[\left(1-A_{d}\right) A_{n d}\right]$ increases. Thus, the two effects could operate in conjunction under some circumstances.

Until now, we have only discussed what we will refer to as positive evidence strategies, under which subjects either actively monitor or search memory for specific types of content (strategic monitoring hypothesis) or apply simple similarity or availability heuristics for recovered nondiagnostic content (IMM model). However, there are source memory phenomena that represent a third possible strategy - for example, the it-had-to-be-you effect (Johnson, Raye, Foley, $\&$ Foley, 1981). This effect reflects the tendency of observers to preferentially misattribute new items to "external" sources during three-choice source monitoring paradigms (e.g., Source A, Source B, or new?). For example, when given a choice between having personally generated the item or having instead had it presented by another person, observers tend to misattribute new items to the external agent. This presumably occurs because the item, although somewhat familiar, is unlikely to evoke contextual memory evidence for prior cognitive operations, which would be likely if it had in fact been personally generated. Given this absence of cognitive memory information in the memory trace, observers infer that it must have come from the source similarly devoid of such cognitive operations - namely, the external one. This effect, along with subjective memorability and distinctiveness heuristics, forms an array of negative evidence strategies, whereby subjects use the absence of a particular type of memory content to inform decision making. Of course, such strategies require that subjects believe that items from one of the sources will highly reliably evoke distinctive and exclusive memory evidence, since otherwise the absence of such evidence would not be particularly informative. As with the strategic monitoring account, however, it is unclear how a negative evidence strategy would be capable of quantitatively linking the simple item recogni- tion differences, item recognition levels, and cue-framing accuracy effects observed here.

In contrast, the IMM model does speak to this relationship and is likely applicable in situations in which the retrieval query is framed in a specifically confirmatory manner (e.g., "Source A?"). This is because the heuristic assumes that item memories that are generally consistent with the prior event description bias subjects toward affirmation when trying to confirm a particular source held in mind. When observers are instead actively encouraged to consider the relative likelihood of two sources, and/or they have a high expectancy that at least one of the sources' items will consistently trigger specific types of distinctive recollections, other decision strategies emphasizing the qualitative nature of the mnemonic differences across queried sources are clearly germane and may operate in conjunction with or in lieu of the IMM heuristic.

\section{Importance for the One-Dimensional Signal Detection Model}

As noted by Marsh and Hicks (1998), the cue-framing accuracy effect during source memory challenges a simple unidimensional signal detection model of this judgment task (see, e.g., Figure 1). This is because, although changing the framing of the cue might lead one to predict a change in response bias estimates, it seems unlikely that detection theorists would have predicted changes in accuracy given this simple manipulation, for the same reason that they have generally assumed that minor manipulations, such as changing the number of reported confidence levels, will not affect measured recognition accuracy. However, one reviewer of the present work did point out that such changes could be easily effected in unidimensional global matching models (Clark \& Gronlund, 1996) by modifying the cue features used to interrogate the global memory architecture. Although this is true, that solution would be somewhat unsatisfying, because such adjustments are apparently unnecessary for item recognition tasks, which instead appear to yield comparable accuracy despite changes in the response cue format (old/new vs. confidence ratings) and framing (e.g., "Old?" vs. "New?" frames). Thus, a full account should explain not only the presence of cue-framing effects during source memory, but also the absence of such effects during item memory. The IMM model does so easily, because the opposition of diagnostic and nondiagnostic memory content is eliminated during simple item recognition judgments in which all recovered content is unambiguously mapped to positive responding. Whether the IMM model is consistent with more complex multidimensional signal detection frameworks (e.g., Banks, 2000) remains to be examined; however, our overall findings suggest that the unidimensional model is an inadequate description of source memory when item recognition differs between sources.

\section{Extensions of the IMM Model}

The IMM model has considerable heuristic value, leading to interesting and clearly testable predictions in other domains. For example, should the cue-framing effect be amplified or lessened in older adult populations? To the ex- 
tent that aging produces an overreliance on gist-level item memory (Koutstaal \& Schacter, 1997) or a disproportionate loss of highly specific diagnostic source memory, Equations 1-4 suggest that the effect should be as, if not more, reliably expressed in older adults, because the expression of nondiagnostic content is what drives the phenomenon. This prediction is interesting because it would serve as another way to confirm that the effect reflects the relatively uncontrolled use of item memory during confirmatory source judgment. In contrast, if the cue-framing effect solely reflects the adoption of a more controlled retrieval strategy, then one might expect a reduced effect in older adults, because they are often argued to show impairments in self-initiated strategic processing (Naveh-Benjamin, Craik, Guez, \& Kreuger, 2005). Thus, whereas the basic IMM model suggests an amplified cue-framing effect in this population, other accounts might arguably suggest a reduction in the effect. Similar reasoning would apply to manipulations of response speeding in young adults. Namely, if speeding a response limits the relative contribution of diagnostic memory more than the availability of nondiagnostic item memory, then under the IMM model, speeding subjects should also amplify the cue-framing relative accuracy effect. Again, this prediction is interesting, because it would reflect a case in which cue-dependent effects would be amplified by the manipulation, whereas one typically expects the ability of subjects to systematically deploy controlled strategies to be minimized under time pressure.

\section{Conclusions}

The present research suggests that subjects use item memory in a heuristic fashion when attempting confirmatory source attributions. This heuristic was formalized into the "item memory misattribution" model, which successfully predicted the quantitative relationship between recognition levels in the items drawn from two sources and the expression of the cue-framing accuracy effect. The IMM model serves as only one of a number of heuristics and strategies that subjects are believed to employ when making source memory decisions, but it is likely an important one, given that individuals may often approach source memory problems in a fairly confirmatory manner. Given the model's successful performance here, further research looking at factors such as aging and response speeding may serve as important tests of its generality.

\section{AUTHOR NOTE}

This work was supported in part by the National Institutes of Health through Grant R01-MH073982 to I.G.D. Correspondence relating to this article may be sent to I. G. Dobbins, Washington University, Department of Psychology, Campus Box 1125, St. Louis, MO 63130 (e-mail: idobbins@wustl.edu).

\section{REFERENCES}

BANKs, W. P. (1970). Signal detection theory and human memory. Psychological Bulletin, 74, 81-99.

BANKs, W. P. (2000). Recognition and source memory as multivariate decision processes. Psychological Science, 11, 267-273.

Clark, S. E., \& Gronlund, S. D. (1996). Global matching models of recognition memory: How the models match the data. Psychonomic Bulletin \& Review, 3, 37-60.

Craik, F. I. [M.], \& LOCKHART, R. S. (1972). Levels of processing: A framework for memory research. Journal of Verbal Learning \& Verbal Behavior, 11, 671-684

Dobbins, I. G., Foley, H., Schacter, D. L., \& Wagner, A. D. (2002). Executive control during episodic retrieval: Multiple prefrontal processes subserve source memory. Neuron, 35, 989-996.

Dodson, C. S., \& Johnson, M. K. (1993). Rate of false source attributions depends on how questions are asked. American Journal of Psychology, 106, 541-557.

Gilovich, T., Griffin, D., \& Kahneman, D. (Eds.) (2002). Heuristics and biases: The psychology of intuitive judgement. Cambridge: Cambridge University Press.

HupPert, F. A., \& Piercy, M. (1976). Recognition memory in amnesic patients: Effect of temporal context and familiarity of material. Cortex, 12, 3-20.

Huppert, F. A., \& Piercy, M. (1978). The role of trace strength in recency and frequency judgements by amnesic and control subjects. Quarterly Journal of Experimental Psychology, 30, 347-354.

JACOBY, L. L. (1991). A process dissociation framework: Separating automatic from intentional uses of memory. Journal of Memory \& Language, 30, 513-541.

JacoBy, L. L., Kelley, C., Brown, J., \& JaSeChKo, J. (1989). Becoming famous overnight: Limits on the ability to avoid unconscious influences of the past. Journal of Personality \& Social Psychology, 56, 326-338.

JaCoBy, L. L., Woloshyn, V., \& Kelley, C. (1989). Becoming famous without being recognized: Unconscious influences of memory produced by dividing attention. Journal of Experimental Psychology: General, 118, 115-125.

Johnson, M. K., Hashtroudi, S., \& Lindsay, D. S. (1993). Source monitoring. Psychological Bulletin, 114, 3-28.

Johnson, M. K., Raye, C. L., Foley, H. J., \& Foley, M. A. (1981). Cognitive operations and decision bias in reality monitoring. American Journal of Psychology, 94, 37-64.

Kelley, R., \& WiXTED, J. T. (2001). On the nature of associative information in recognition memory. Journal of Experimental Psychology: Learning, Memory, \& Cognition, 27, 701-722.

Koriat, A., \& LEVY-SADOT, R. (2001). The combined contributions of the cue-familiarity and accessibility heuristics to feelings of knowing. Journal of Experimental Psychology: Learning, Memory, \& Cognition, 27, 34-53.

KoutstaAl, W., \& Schacter, D. L. (1997). Gist-based false recognition of pictures in older and younger adults. Journal of Memory \& Language, 37, 555-583.

Lindsay, R. C. L., Pozzulo, J. D., Craig, W., Lee, K., \& Corber, S. (1997). Simultaneous lineups, sequential lineups, and showups: Eyewitness identification decisions of adults and children. Law \& Human Behavior, 21, 391-404.

Macmillan, N. A., \& Creelman, C. D. (1991). Detection theory: A user's guide. Cambridge: Cambridge University Press.

MARSH, R. L., \& HicKs, J. L. (1998). Test formats change sourcemonitoring decision processes. Journal of Experimental Psychology: Learning, Memory, \& Cognition, 24, 1137-1151.

Naveh-Benjamin, M., Craik, F. I. M., Guez, J., \& Kreuger, S. (2005). Divided attention in younger and older adults: Effects of strategy and relatedness on memory performance and secondary task costs. Journal of Experimental Psychology: Learning, Memory, \& Cognition, 31, 520-537.

NiCKERSON, R. S. (1998). Confirmation bias: A ubiquitous phenomenon in many guises. Review of General Psychology, 2, 175-220.

PARKS, C. M. (2007). The role of noncriterial recollection in estimating recollection and familiarity. Journal of Memory \& Language, 57, 81-100.

PARKS, T. E. (1966). Signal-detectability theory of recognition-memory performance. Psychological Review, 73, 44-58.

Qin, J., Raye, C. L., Johnson, M. K., \& Mitchell, K. J. (2001). Source ROCs are (typically) curvilinear: Comment on Yonelinas (1999). Journal of Experimental Psychology: Learning, Memory, \& Cognition, 27, 1110-1115.

SLOTNICK, S. D., \& Dodson, C. S. (2005). Support for a continuous (single-process) model of recognition memory and source memory. Memory \& Cognition, 33, 151-170.

Whittlesea, B. W., Jacoby, L. L., \& Girard, K. (1990). Illusions of immediate memory: Evidence of an attributional basis for feelings of familiarity and perceptual quality. Journal of Memory \& Language, 29, 716-732. 


\section{NOTES}

1. Although the false alarm rates for new items fluctuated, the ability to discriminate between the two classes of old items (seen and heard) was unaffected by the framing of the cue.

2. The hits-minus-false-alarms accuracy metric implies a threshold discrimination process that is poorly supported in the case of receiver operating characteristics (ROCs) for simple item recognition data, and its appropriateness for characterizing source memory ROCs remains a matter of debate. Here we chose this metric because of its simplicity and its monotonic relationship with other accuracy metrics, and for ease of exposition. In addition, the metric has the further advantage of being defined at extreme response rates.

3. Equations 1-4 can also be used to estimate the relative diagnostic and nondiagnostic content levels of the two item types, in a manner similar to how recollection and familiarity are estimated in the process dissociation procedure (Jacoby, 1991). However, the validity of the estimates critically relies on the stability of the "yes" response criterion across the cue frames. If the criterion remains fixed, the difference between Equations 1 and 4 specifies the proportion of diagnostic information available for $A$ items $\left(A_{d}\right)$. Given this value, one could then use either Equation 1 or 4 to calculate the level of nondiagnostic information for $A$ items $\left(A_{n d}\right)$. The analogous values for the B items would be calculated in a similar manner, on the basis of the difference between Equations 3 and 2. These diagnostic and nondiagnostic memory values under the IMM model do not, however, correspond to process-pure measures of recollection and fa- miliarity. Furthermore, given that the response rates to new items changed under the two source cue frames in Experiment 1, and that similar shifts are noted in Marsh and Hicks (1998), the critical assumption of criterion stability across the cue frames is likely not met in these paradigms.

4. The values in Equations 1 and 3 need not reflect the level of absolute recognition that would be observed were the items to be tested in a simple recognition task, and indeed observers might adopt a more liberal criterion when asked to detect these items during simple recognition testing. However, the critical value for the predictions is based on the difference in the rates from the equations (i.e., A - B). Thus, the assumption of the model is that this difference will reliably covary with differences in the endorsement rates of the same items when seen during simple recognition testing.

5. We use the terminology of model confirmations and violations for ease of exposition. Strictly speaking, the failure to observe cue-framing accuracy effects that are concordant with observed recognition differences does not constitute a failure or violation of the model's predictions, because the model in fact specifies that this should increasingly occur under certain conditions - namely, low recognition difference levels and high absolute recognition levels. That is, the model actually specifies the relative likelihoods of observing cue-framing accuracy effects that are concordant versus discordant with the direction of observed recognition differences. We simply use confirmations and violations to denote these tendencies because the terminology is less cumbersome than more precise alternatives.

(Manuscript received November 8, 2006; revision accepted for publication July 17,2007 .) 